\title{
Petro-geochemical characteristics and origin of the quartz in the lower oceanic crust, example from IODP- Hole U1473A
}

\author{
Du Khac Nguyen ${ }^{1, *}$ Tomoaki Morishita ${ }^{2}$ \\ 1 Faculty of Geosciences and Geoengineering, Hanoi University of Mining và Geology, Vietnam \\ 2 Kanazawa University, Kakuma, Kanazawa, Ishikawa 920-1192,Japan
}

\begin{abstract}
ARTICLE INFO ABSTRACT
Article history:

Received $12^{\text {th }}$ May 2020

Revised 29th July 2020

Accepted 31stAug. 2020

Keywords:

Crystallization,

Felsic vein,

Lower gabbro,

Quartz,

Repricipitation.

IODP-Hole U1473A was drilled on the summit of Atlantis bank, Southwest Indian Ridge recovered large amounts of gabbroic rocks including mainly olivine gabbro. Felsic rocks are minor, approximately 1,5\% of the total volume, which are comprising significant amount of quartz in some samples. The Ti concentrations and the estimated temperatures of the quartz in veins are relatively high, ranging from $30 \div 130 \mathrm{ppm}$ and $540 \div 700^{\circ} \mathrm{C}$, coupled with the myrmekitic textures in some veins are unambigeous evidence for the late magmatic origin. In addition to the crystallization mechanism in free spaces, such as crack/ fracture systems during the penetration of $\mathrm{SiO}_{2}$ - saturated magmas; the quartz is also formed by re-precipitation process at the same location leaving behind after the previous olivine in the host gabro has been dissolved.
\end{abstract}

Copyright @ 2020 Hanoi University of Mining and Geology. All rights reserved.

${ }^{*}$ Corresponding author

E-mail: nguyenkhacdu@humg.edu.vn

DOI: 10.46326/ JMES.2020.61(4).07 


\title{
Tạp chí Khoa học Kỹ thuật Mỏ - Địa chất
}

Trang điện tử: http://tapchi.humg.edu.vn

\section{Luận bàn về đặc điểm, nguồn gốc và cơ chế thành tạo khoáng vật thạch anh của đai mạch felsic trong lớp vỏ dưới gabro thuộc kiểu vỏ đại dương thực thụ, ví dụ từ lỗ khoan U1473A}

\author{
Nguyễn Khắc Du ${ }^{1, *}$, Tomoaki Morishita ${ }^{2}$ \\ ${ }^{1}$ Khoa Khoa học và Kỹ thuật Địa chất, Trường Đại học Mỏ - Địa chất, Việt Nam \\ 2 Trường Đại học Kanazawa, Ishikawa, Nhật Bản
}

\section{THÔNGTIN BÀI BÁO TÓMTẮT}

\section{Quá trình:}

Nhận bài 12/05/ 2020

Sửa xong 29/07/2020

Chấp nhận đăng 31/ 08/ 2020

\section{Tù̀ khóa:}

Đai mạch felsic

/ plagiogranit,

Kết tinh,

Thạch anh,

Tái lắng đọng,

Vỏ gabro.
Công trình khoan U1473A được thực hiện trên đỉnh núi ngầm Atlantis dưới đáy Ân Độ Dương đã thu hồi lương lớn mẫu lỗi khoan gồm chủ yếu các đá thuộc chuỗi gabro có thành phần tù̀ gabro olivin đến gabro chứa oxit. Các đai mạch có thành phần felsic chỉ chiếm lương nhỏ, khoảng 1,5\% tổng lượng mẫu nhưng có ý nghĩa lớn trong nghiến cúu tiến hóa magma sống núi giữa đại dương. Kết quả phân tích thanh phần thạch học và đặc điểm địa hóa đã chỉ ra rằng khoáng vật thạch anh trong các đai mach này khá giàu thành phần Ti $(30 \div 130 \mathrm{ppm})$ và có nhiệt độ thành tạo tương đối cao $\left(540 \div 700^{\circ} \mathrm{C}\right)$, kèm theo vi kiến trúc khảm myrmekit/ granophyr là bằng chứng rất rõ ràng cho nguồn gốc magma muộn của các đai mạch felsic/ plagiogranit. Nghiên cúu cho thấy các khoáng vật thạch anh này có thể là sản phẩm kết tinh trực tiếp tại các khe nút, đứt gãy trong quá trinh xâm nhâp của dung thể magma giàu thành phần $\mathrm{SiO}_{2}$ vào lớp vỏ gabro, tuy nhiên chúng cũng có thể là sản phẩm tái lắng đọng kết tinh tại vị trí của khoáng vật olivin trong các đá gabro olivin thuộc lớp vỏ đại dương thực thu.

(C) 2020 Trường Đại học Mỏ - Địa chất. Tất cả các quyền được bảo đảm.

\section{Mở đầu}

Lỗ khoan U1473A thuộc Chương trình Quốc tế khám phá đại dương (IODP) đã khoan được gần $800 \mathrm{~m}$ trên đỉnh núi ngầm Atlantis, sống núi tây nam Ấn Độ Dương. Tập mẫu lõi khoan gồm khối lượng lớn các đá thuộc chuỗi gabro có thành phần

\section{*Tác giả liên hệ}

E - mail: nguyenkhacdu@humg.edu.vn

DOI: 10.46326/ JMES.2020.61(4).07 chủ yếu là gabro olivin, ít hơn là các đá gabro, gabro chứa oxit và các đai mạch felsic. Trong đó, khối lượng các đai mạch felsic/ plagiogranit chỉ chiếm khoảng 1,5\% tổng lượng mẫu (MacLeod và nnk., 2017), tuy nhiên chúng xuất hiện khá phổ biến trong lõi khoan và thường dễ dàng được phân biệt với các đá gabro bằng mắt thường.

Cho đến nay, nhiều nhà địa chất cho rằng các đai mạch felsic/ plagiogranit có thể có nguồn gốc magma thực thụ hoặc cũng có thể là sản phẩm nhiệt dịch/ hậu magma. Trong trường hợp các đai mạch này là sản phẩm của quá trình magma thực 
thụ, có ít nhất 3 mô hình có thể giải thích cho sự thành tạo dung thể giàu $\mathrm{SiO}_{2}$ như sau: (1) Nóng chảy từng phần các đá biến đổi (Koepke và nnk., 2004, 2007; Koepke., 2005); (2) kết tinh phân đoạn (Niu và nnk., 2002); và (3) magma dung ly (Charlier, Grove, 2012; Dixon và Rutherford, 1979; Philpotts, 1979). Do đó, việc khẳng định nguồn gốc cũng như cơ chế thành tạo các đai mạch này có ý nghĩa lớn trong nghiên cứu bản chất quá trình tiến hóa của magma bên dưới sống núi giữa đại dương (MORB).

Các đai mạch felsic/ plagiogranit có thành phần chủ yếu gồm plagioclas, amphibol nâu đỏ, các oxit Fe và Ti, thhạch anh. Khoáng vật phụ gồm zircon, apatit, titanit, \#biotit, \#felspat-K. Các khoáng vật thứ sinh gồm amphibol lục, \pm cacbonat, \pm sét. Theo bảng phân loại đá sâu của Hiệp hội Địa chất Quốc tế (IUGS) được đề xuất bởi (Le Maitre và nnk., 2002), các đai mạch felsic/ plagiogranit này là hornblend gabro, anorthosit chứa hornblend và anorthosit chứa thạch anh theo nghĩa hẹp. Trên biểu đồ Q-A-P (thạch anh - felspat kali plagioclas), các đai mạch có thành phần felsic bao gồm diorit, diorit thạch anh và tonalit.

Thạch anh là khoáng vật kết tinh cuối cùng trong chuỗi phản ứng Bowen, tuy nhiên, khoáng vật này cũng có thể được lắng đọng từ các dung dịch nhiệt dịch hậu magma nhiệt độ thấp đến trung bình. Vì vậy việc nghiên cứu, phân biệt, làm sáng tỏ các loại hình nguồn gốc, cơ chế thành tạo khoáng vật này đóng vai trò quan trọng trong việc luận giải nguồn gốc các đai mạch felsic, làm cơ sở nghiên cứu quá trình tiến hóa magma sống núi giữa đại dương. Trong công trình này, dựa vào đặc điểm thạch học và thành phần địa hóa khoáng vật thạch anh trong các đai mạch, tác giả sẽ luận giải về nguồn gốc, điều kiện và cơ chế thành tạo của chúng, từ đó góp phần hiểu biết hơn về nguồn gốc các đai mạch felsic/ plagiogranit nêu trên.

\section{Phương pháp nghiên cứu}

\subsection{Phương pháp nghiên cúu thành phần khoáng vật}

Để nghiên cứu đặc điểm khoáng vật các đá khu vực núi ngầm Atlantis, trung tâm tách giãn tây nam Ấn Độ Dương, phương pháp được sử dụng gồm phân tích thạch học lát mỏng dưới kính hiển vi phân cực kết hợp với đo vẽ X-ray trên thiết bị M4 - Tornado, Bruker tại Viện Khoa học Côngnghệ
Địa chất Nhật Bản. Các phép đo sử dụng dòng điện $50 \mathrm{kV}$ với cường độ $600 \mu \mathrm{A}$; chùm tia $\mathrm{X}$ có đường kính là $25 \mu \mathrm{m}$, mỗi điểm ảnh được đo vẽ có kích thước 20 x $20 \mu \mathrm{m}$ trong thời gian 1 mili giây. Sau đó, sử dụng phần mềm ImageJ, các bức ảnh riêng lẻ của từng nguyên tố được chồng ghép tạo ra ảnh phân bố các khoáng vật trên toàn bộ mẫu hoặc được sử dụng trực tiếp để phục vụ tính toán định lượng thành phần thạch học các đá.

\subsection{Phương pháp nghiên cứu địa hóa khoáng vật}

Thành phần chính và vết của thạch anh được xác định bằng thiết bị vi phân tích hiển vi đầu dò (EPMA) JEOLJXA - 8800 sử dụng phần mềm hiệu chỉnh ZAF tại Đại học Kanazawa, Nhật Bản. Các phân tích được thực hiện trong điều kiện điện áp $20 \mathrm{kV}$, chùm tia electron có cường độ $20 \mathrm{nA}$, đường kính $3 \mu \mathrm{m}$. Tại các vị trí điểm cao và phông nền của tia $X$, nguyên tố $S i$ được phân tích lần lượt trong 20 giây và 10 giây. Trong khi đó, để thu được hàm lượng các nguyên tố $\mathrm{Al}, \mathrm{K}, \mathrm{Fe}$, Ti có độ chính xác cao, tác giả đã áp dụng phương pháp phân tích đề xuất bởi (Müller và nnk., 2003) trong thời gian lâu hơn (300 giây và 150 giây). Các mẫu chuẩn quốc tế bao gồm mẫu tự nhiên và mẫu tổng hợp có thành phần đã được công bố trên các tạp chí quốc tế uy tín.

\section{Kết quả và thảo luận}

\section{1. Đặc điểm thạch học}

Trong một số đai mạch felsic, thạch anh xuất hiện với hàm lượng đáng kể $(3 \div 25 \%)$ dưới các dạng sau: (1) Mọc ghép với plagioclas tạo thành kiến trúc myrmekit/ granophyr; (2) Dạng hạt nhỏ tha hình, tàn dư lấp đầy lỗ hổng giữa các khoáng vật thành tạo trước, dao động từ $0,1 \div 0,5 \mathrm{~mm}$ hoặc hiện diện giữa các đai mạch thành phần felsic với cỡ hạt lớn hơn (đến 2 mm) (Hình 1). Bên cạnh đó, trong tập mẫu nghiên cứu khá phổ biến, hiện tượng các hạt thạch anh có ranh giới rõ ràng nằm sát cạnh nhau nhưng có đặc tính quang học liên tục, tạo thành tập hợp hạt lớn (đến >3mm).

\section{2. Đặc điểm địa hóa}

Theo kết quả phân tích EPMA, các hạt khoáng vật thạch anh trong tập mẫu nghiên cứu có thành phần $\mathrm{SiO}_{2}$ dao động từ $98,795 \div 101,351 \%$. Để 

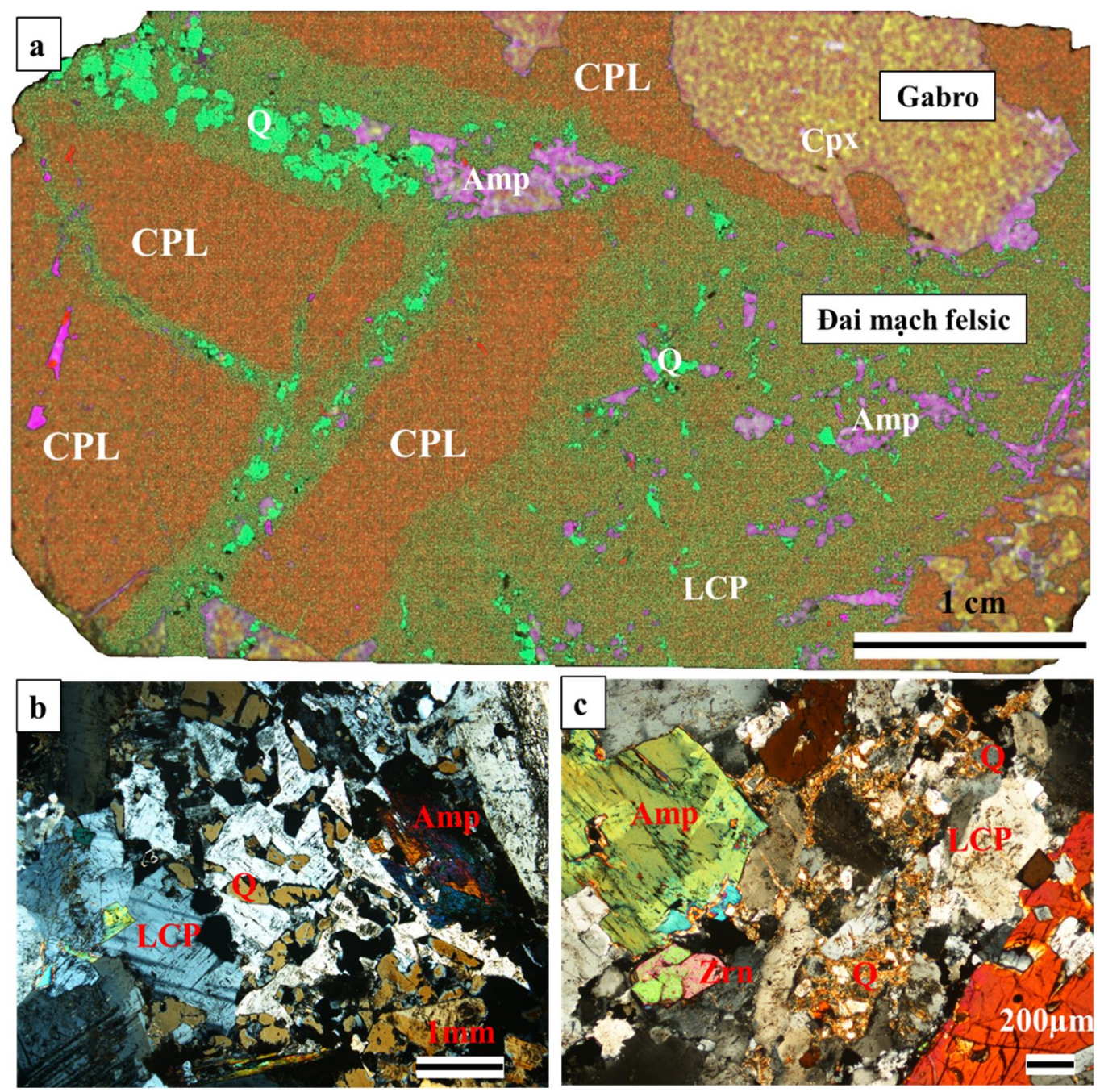

Hình 1. (a) Ảnh đo vẽ X-ray của toàn bộ mẫu lát mỏng (Mẫu 66R4, 46-49cm). Kết quả cho thấy mẫu gồm 2 loại đá có thành phần khác nhau: (1) gabro chúa plagioclas giàu calci+pyroxen xiên,

(2) các đai mạch felsic/ plagiogranit chúa plagioclas nghèo calci+Amp. Các dạng tồn tại của thạch anh trong các đai mạch felsic gồm: (b) cộng sinh cùng thạch anh tạo ra kiến trúc myrmekit (Mẫu 64R2, 110-113 cm) và (c) lấp đầy các kẽ hở giữa các khoáng vật kết tinh trước (Mẫu 64R2, 128-133 cm). (Cpx: Pyroxen xiên. CPL: Plagioclas giàu calci. LCP: Plagioclas nghèo calci. Amp: Amphibol. Q: thạch anh. Zrn: Zircon).

kiểm tra thành phần nguyên tố vết (gồm Ti, $K, \mathrm{Fe}$, và $\mathrm{Al}$ ), tác giả đã lựa chọn phân tích 26 hạt khoáng vật trong 4 mẫu thạch học lát mỏng, trong đó một mẫu $(64 R 2,110 \div 113 \mathrm{~cm})$ có chứa khoáng vật thạch anh dưới dạng mọc ghép với khoáng vật plagioclas tạo thành kiến trúc khảm myrmekit/ granophyr rất đặc trưng. Trong các mẫu khác, thạch anh tồn tại dạng tàn dư lấp đầy các lỗ hổng $(<0,2 \mathrm{~mm})$ và/ hoặc tạo thành các tập hợp hạt lớn hơn (> $1 \mathrm{~mm}$ ) trong 2 mẫu còn lại (Mẫu 64R2, $128 \div 133 \mathrm{~cm}$; $66 \mathrm{R} 5,1 \div 7 \mathrm{~cm}$ và $66 \mathrm{R} 4,46 \div 49 \mathrm{~cm})$.
Kết quả phân tích thành phần các nguyên tố vết trong thạch anh được thể hiện trong Hình 2, tất cả các hạt thạch anh được phân tích cho kết quả hàm lượng Ti và $K$ tương đối cao, dao động lần lượt từ $30 \div 130$ ppm và $20 \div 60$ ppm. Hàm lượng Fe biến đổi mạnh trong thạch anh, dao động từ bên dưới giới hạn phân tích của thiết bị đến 180 ppm. Trong khi đó, thành phần $\mathrm{Al}$ dưới giới hạn phân tích của EPMA trong tất cả các mẫu phân tích. Điểm nổi bật nhất từ các kết quả phân tích trên là các hạt thạch anh tồn tại dưới dạng tàn dư, lấp đầy lỗ hổng giữa các khoáng vật khác có thành phần tương tự với 

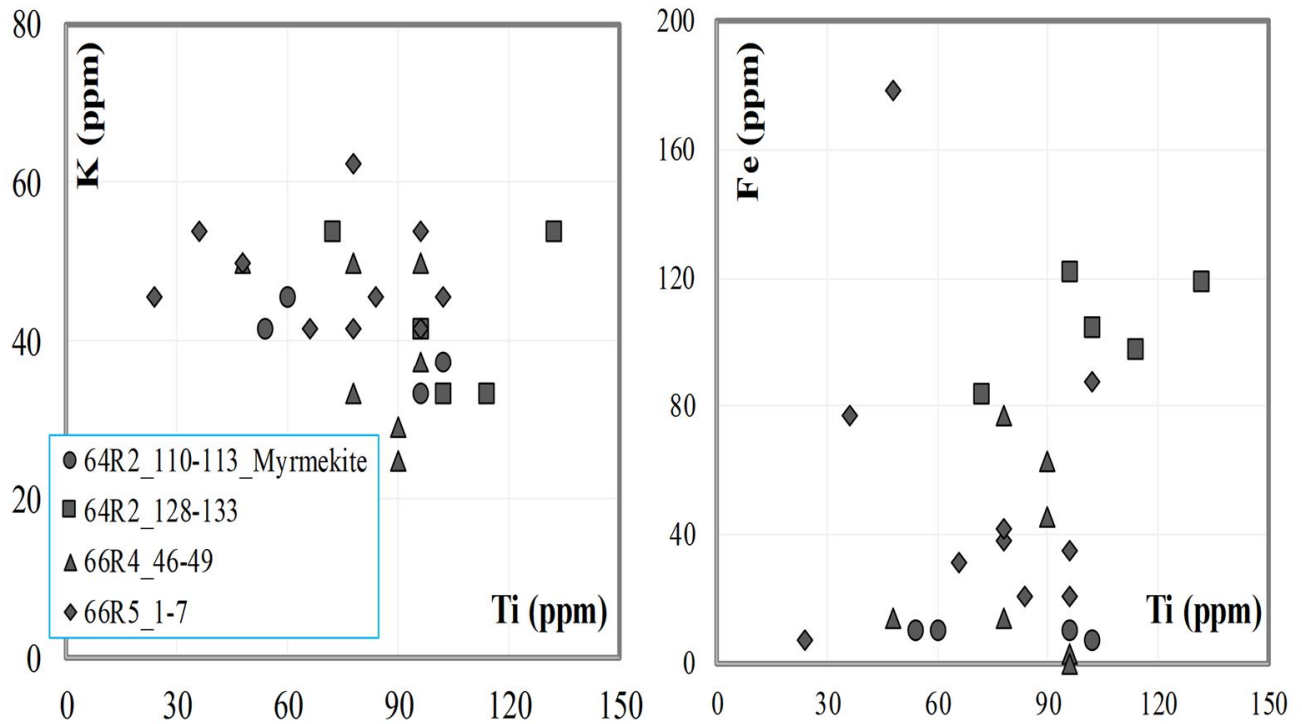

Hình 2. Tương quan hàm lượng các nguyên tố vêt (a) K-Ti và (b) Ti-Fe của khoáng vật thạch anh trong các đai mạch felsic/ plagiogranit khu vực núi ngầm Atlantis, trung tâm tách giãn tây nam Ấn Độ Dương.

các hạt thạch anh tồn tại dưới dạng mọc ghép với plagioclas (myrmekit/ granophyr).

\subsection{Nhiệt độ thành tạo khoáng vật thạch anh}

Nhiệt độ cân bằng của thạch anh trong các đai mạch felsic/ plagiogranit được tính toán dựa vào hàm lượng Ti trong khoáng vật này. Đây là nhiệt kế khoáng vật thạch anh lần đầu tiên được biết đến với tên gọi "nhiệt kế TitaniQ", sau đó được hiệu chỉnh chính xác hơn trong công trình nghiên cứu của Huang và Audestat. (2012). Trong đó, giá trị áp suất được sử dụng là 1 kbar thu được từ kết quả tính toán cho khoáng vật amphibol màu nâu đỏ trong các mẫu này (không trình bày chi tiết ở đây). Các kết quả tính toán cho thấy, khoáng vật thạch anh trong các đai mạch felsic khu vực Atlantis có nhiệt độ cân bằng khá cao, dao động $540 \div 700{ }^{\circ} \mathrm{C}$ (Bảng 1$)$.

\subsection{Nguồn gốc và cơ chế thành tạo khoáng vật thạch anh}

Thạch anh trong các đai mạch felsic có thể là sản phẩm kết tinh từ dung thể magma bão hòa $\mathrm{SiO}_{2}$ hoặc là sản phẩm lắng đọng từ các dung dịch nhiệt dịch. Để phân biệt được các loại nguồn gốc khác nhau của khoáng vật này, tác giả dựa vào đặc điểm cấu tạo - kiến trúc dưới kính hiển vi và hàm lượng Ti cũng như điều kiện nhiệt độ thành tạo của khoáng vật. Vì thạch anh tồn tại dưới dạng mọc ghép với plagioclas (kiến trúc myrmekit/ granophyr) (Hình 1) đã được chấp nhận rộng rãi là sản phẩm kết tinh ở nhiệt độ cao/ magma thực thụ, thành phần hóa học của chúng được sử dụng để so sánh với thành phần của thạch anh trong các mẫu khác. Bên cạnh đó, nhiều tác giả cũng đã chỉ ra rằng hàm lượng Ti là chỉ thị rất hữu ích để phân biệt các loại hình nguồn gốc khác nhau của khoáng vật này. Trong đó, thạch anh có nguồn gốc magma thực thụ sẽ chứa hàm lượng Ti đáng kể, nhưng lại rất thấp (thường $<5$ ppm) trong các hạt thạch anh có nguồn gốc nhiệt dịch (Breiter và nnk., 2017; Jacamon và Larsen, 2009; Müller và nnk., 2003).

Kết quả nghiên cứu địa hóa cho thấy, các phân tích đều cho hàm lượng Ti khá cao $(30 \div 130$ ppm) trong tất cả các hạt thạch anh dưới dạng myrmekit hoặc dạng lấp đầy lỗ hổng, tương ứng với nhiệt độ thành tạo tương đối cao $\left(540 \div 700^{\circ} \mathrm{C}\right)$ của khoáng vật này (Hình 2, Bảng 1). Nhiệt độ này hoàn toàn phù hợp với giai đoạn magma muộn và không hề có liên quan về mặtnguồn gốc đến hoạt động nhiệt dịch hậu magma. Vì vậy, tác giả đưa ra kết luận rằng khoáng vật thạch anh là sản phẩm kết tinh từ dung thể magma muộn bão hòa $\mathrm{SiO}_{2}$.

Bên cạnh đó, thạch anh và các tập hợp khoáng vật ẩn tinh hoặc vô định hình giàu $\mathrm{Fe}, \mathrm{Mg}$ trong một số đai mạch felsic có tính tương đồng về khối lượng, kích cỡ và hình dạng so với các khoáng vật olivin trong đá gabro (Hình 3). Do đó, tác giả đề xuất rằng khoáng vật olivin trong đá gốc gabro đã 
Bảng 1. Tổng hợ kết quả phân tích, tính toán nhiệt độ thành tạo của khoáng vật thạch anh trong các đai mạch plagiogranit khu vực Atlanstis, Ấn Độ Dương theo công thức của Huang và Audétat (2012) tại áp suất 1 kbar.

\begin{tabular}{|c|c|c|c|c|c|c|c|c|c|c|}
\hline $\begin{array}{c}\text { IODP- } \\
\text { LK. } \\
\text { U1473A }\end{array}$ & TT & $\begin{array}{l}\text { Số hiệu điểm } \\
\text { Phân tích }\end{array}$ & $\begin{array}{l}\mathrm{SiO}_{2} \\
(\%)\end{array}$ & $\begin{array}{c}\mathrm{K} \\
(\mathrm{ppm})\end{array}$ & $\begin{array}{c}\mathrm{Ti} \\
(\mathrm{ppm})\end{array}$ & $\begin{array}{c}\mathrm{Fe} \\
(\mathrm{ppm})\end{array}$ & $\begin{array}{c}\mathrm{T} \\
\left({ }^{\circ} \mathrm{C}\right)\end{array}$ & $\begin{array}{l}\text { Trung } \\
\text { bình }\end{array}$ & Min & Max \\
\hline \multirow{4}{*}{$\begin{array}{c}64 \mathrm{R} 2, \\
110 \div 113 \\
\mathrm{~cm}\end{array}$} & 1 & 64R2W_110-113_Q1 & 101,096 & 46 & 60 & 10 & 620 & \multirow{4}{*}{644} & \multirow{4}{*}{610} & \multirow{4}{*}{677} \\
\hline & 2 & 64R2W_110-113_Q2 & 99,635 & 37 & 102 & 7 & 677 & & & \\
\hline & 3 & 64R2W_110-113_Q3 & 99,992 & 33 & 96 & 10 & 670 & & & \\
\hline & 4 & 64R2W_110-113_Q4 & 100,874 & 42 & 54 & 10 & 610 & & & \\
\hline \multirow{5}{*}{$\begin{array}{c}64 \mathrm{R} 2, \\
128 \div 133 \\
\mathrm{~cm}\end{array}$} & 5 & 64R2W_128-133_Q1 & 99,223 & 54 & 132 & 119 & 707 & \multirow{5}{*}{676} & \multirow{5}{*}{639} & \multirow{5}{*}{707} \\
\hline & 6 & 64R2W_128-133_Q2 & 99,74 & 33 & 102 & 105 & 677 & & & \\
\hline & 7 & 64R2W_128-133_Q3 & 100,79 & 54 & 72 & 84 & 639 & & & \\
\hline & 8 & 64R2W_128-133_Q4 & 100,465 & 33 & 114 & 98 & 689 & & & \\
\hline & 9 & 64R2W_128-133_Q5 & 100,428 & 42 & 96 & 122 & 670 & & & \\
\hline \multirow{7}{*}{$\begin{array}{c}66 \mathrm{R} 4 \\
46 \div 49 \\
\mathrm{~cm}\end{array}$} & 10 & 66R4W_46-49_Q1 & 99,493 & 37 & 96 & 3 & 670 & \multirow{7}{*}{651} & \multirow{7}{*}{598} & \multirow{7}{*}{670} \\
\hline & 11 & 66R4W_46-49_Q2 & 101,351 & 50 & 48 & 14 & 598 & & & \\
\hline & 12 & 66R4W_46-49_Q3 & 101,012 & 50 & 96 & 0 & 670 & & & \\
\hline & 13 & 66R4W_46-49_Q4 & 101,012 & 50 & 78 & 77 & 647 & & & \\
\hline & 14 & 66R4W_46-49_Q5 & 99,097 & 33 & 78 & 14 & 647 & & & \\
\hline & 15 & 66R4W_46-49_Q6 & 99,047 & 29 & 90 & 63 & 663 & & & \\
\hline & 16 & 66R4W_46-49_Q7 & 98,795 & 25 & 90 & 45 & 663 & & & \\
\hline \multirow{10}{*}{$\begin{array}{l}66 \mathrm{R} 5 \\
1 \div 7 \mathrm{~cm}\end{array}$} & 17 & 66R5W_1-7_Q1 & 98,903 & 42 & 66 & 31 & 630 & \multirow{10}{*}{630} & \multirow{10}{*}{537} & \multirow{10}{*}{677} \\
\hline & 18 & 66R5W_1-7_Q2 & 99,761 & 54 & 36 & 77 & 572 & & & \\
\hline & 19 & 66R5W_1-7_Q3 & 100,346 & 50 & 48 & 178 & 598 & & & \\
\hline & 20 & 66R5W_1-7_Q4 & 99,892 & 46 & 84 & 21 & 655 & & & \\
\hline & 21 & 66R5W_1-7_Q5 & 100,525 & 46 & 24 & 7 & 537 & & & \\
\hline & 22 & 66R5W_1-7_Q6 & 99,668 & 42 & 96 & 21 & 670 & & & \\
\hline & 23 & 66R5W_1-7_Q7 & 99,784 & 42 & 78 & 38 & 647 & & & \\
\hline & 24 & 66R5W_1-7_Q8 & 100,278 & 54 & 96 & 35 & 670 & & & \\
\hline & 25 & 66R5W_1-7_Q9 & 100,015 & 46 & 102 & 87 & 677 & & & \\
\hline & 26 & 66R5W_1-7_Q10 & 99,616 & 62 & 78 & 42 & 647 & & & \\
\hline
\end{tabular}

bị hòa tan hoàn toàn, đồng thời với sự thành tạo của plagioclas nghèo calci và amphibol màu nâu đỏ (Nguyen và nnk., 2018) do phản ứng của plagioclas giàu calci và pyroxen trong đá gốc với dung thể magma bão hòa $\mathrm{SiO}_{2}$ trong quá trình thành tạo các đai mạch.

Quá trình tái cân bằng giữa dung thể magma muộn với các đá gabro kết thúc bằng sự kết tinh/ lắng đọng của thạch anh và các tập hợp giàu $\mathrm{Fe}, \mathrm{Mg}$ 

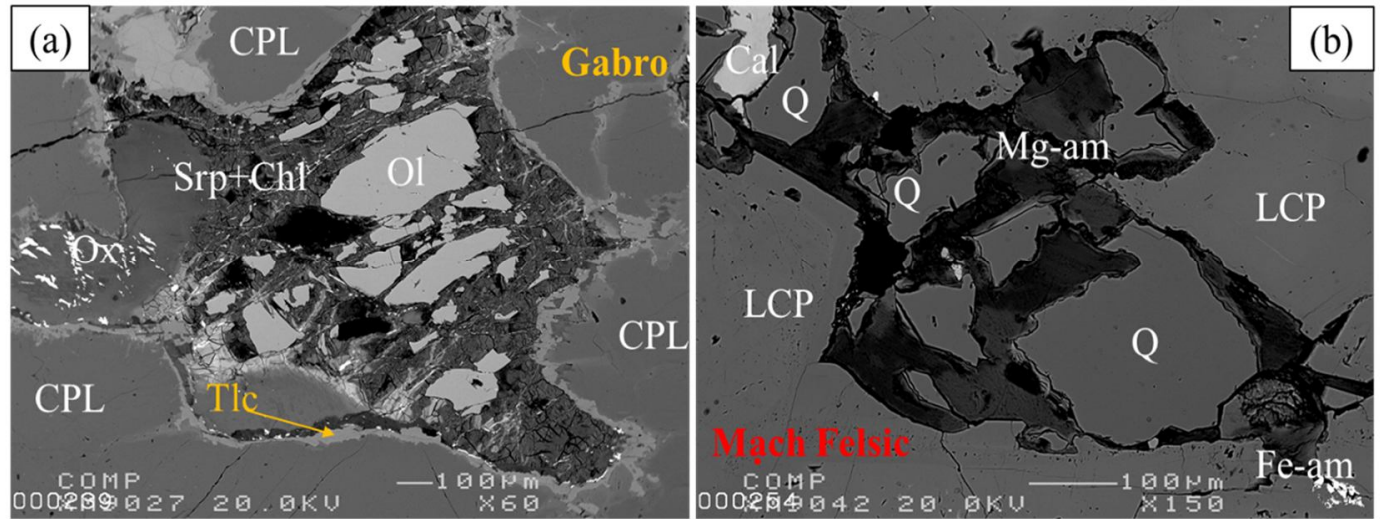

Hình 3. Sự biến đổi của khoáng vật olivin trong gabro: (a) được so sánh với sự xuất hiên của

thạch anh trong đai mạch felsic; (b) mẫu 66R5, 1-7cm. Nguồn: (Nguyen và nnk., 2018).

(CPL: Plagioclas giàu calci. LCP: Plagioclas nghèo calci. Q: Thạch anh. Cal: Calcit. Fe-am:

Khoáng vật ẩn tinh giàu Fe. Mg-am: Khoáng vật ẩn tinh giàu Mg. Sap: Saponit. Ol: Olivin. Srp:

Serpentin. Chl: Chlorit. Tlc: Talc. Ox: Oxit Fe-Ti).

tại đúng vị trí để lại của olivin có trước và được thể hiện thông qua phương trình sau:

$100 \mathrm{~g}$ olivin $(\mathrm{Fo} 76)+38,68 \mathrm{~g} \mathrm{SiO}_{2} \rightarrow 77,06 \mathrm{~g}$ thach anh +39,77 g MgO +21,88 g FeO* +0,3 g MnO

Xét về mặt bản chất, đây là quá trình tái cân bằng giữa đá có trước - dung thể magma có sau với cơ chế hòa tan - tái kết tinh/ lắng đọng (Putnis, 2002, 2009; Putnis và John., 2010; Putnis và Putnis, 2007) diễn ra hết sức phức tạp so với quá trình kết tinh các khoáng vật thông thường.

\section{Kết luận}

Các kết quả nghiên cứu chính của công trình này có thể được tóm gọn lại như sau:

(1) Khoáng vật thạch anh trong các đai mạch felsic tương đối giàu hàm lượng Ti $(30 \div 130$ ppm) và nhiệt độ cân bằng khá cao $\left(540 \div 700^{\circ} \mathrm{C}\right)$, cùng với kiến trúc myrmekit/ granophyr là nhưng minh chứng rõ ràng cho nguồn gốc magma muộn.

(2) Ngoài cơ chế kết tinh trực tiếp trong hệ thống các khe nứt, đứt gãy; các hạt thạch anh trong các đá gabro chứa các đai mạch felsic còn được thành tạo tại vị trí của khoáng vật olivin trong đá gabro olivin có trước.

\section{Lời cảm ơn}

Nghiên cứu sử dụng mẫu lõi khoan thuộc Chương trình Khám phá Đại dương (IODP, Lỗ khoan U1473A, https:// www.iodp.org/ aboutiodp). Công tác gia công, phân tích mẫu được thực hiện tại Trường Đại học Kanazawa, với sự giúp đỡ tận tình của Yumiko Harigane tại Viện Khoa học và Công nghệ Địa chất Nhật Bản trong công tác đo vẽ X-ray. Bên cạnh đó, những trao đổi, thảo luận của tác giả với giáo sư Biswajit Ghosh (Đại học Kolkata, Ấn Độ) rất hữu ích để hoàn thiện công trình này. Kinh phí thực hiện nghiên cứu được Bộ Giáo dục và Đào tạo Việt Nam và Đại học Kanazawa đài thọ. Tác giả xin chân thành cảm ơn tất cả những sự hỗ trợ của các cá nhân, tổ chức nêu trên.

\section{Tài liệu tham khảo}

Breiter, K., Ďurišová, J. and Dosbaba, M., (2017). Quartz chemistry - A step to understanding magmatic-hydrothermal processes in orebearing granites: Cínovec/ Zinnwald Sn-W-Li deposit, Central Europe. Ore Geol Rev. 90, 25-35.

Charlier, B. and Grove, T. L., (2012). Experiments on liquid immiscibility along tholeiitic liquid lines of descent. Contrib. Mineral. Petrol. 164, 27-44.

Dixon, S. and Rutherford, M. J., (1979). Plagiogranites as late-stage immiscible liquids in ophiolite and mid-ocean ridge suites: An experimental study. Earth. Planet. Sci. Lett. 45, $45-60$.

Huang, R. and Audétat, A., (2012). The titaniumin-quartz (TitaniQ) thermobarometer: A critical examination and re-calibration. Geochim. Cosmochim. Acta. 84, 75-89. 
Jacamon, F. and Larsen, R. B., (2009). Trace element evolution of quartz in the charnockitic Kleivan granite, SW-Norway: The Ge/ Ti ratio of quartz as an index of igneous differentiation. Lithos. 107, 281-291.

Koepke, J., Berndt, J., Feig, S. T. and Holtz, F., (2007). The formation of $\mathrm{SiO}_{2}$-rich melts within the deep oceanic crust by hydrous partial melting of gabbros. Contrib. Mineral. Petrol. 153, 67-84.

Koepke, J., Feig, S. and Snow, J., (2005). Late stage magmatic evolution of oceanic gabbros as a result of hydrous partial melting: Evidence from the Ocean Drilling Program (ODP) Leg 153 drilling at the Mid-Atlantic Ridge. Geochem., Geophys., Geosyst. 6, 27 pages.

Koepke, J. r., Feig, S. T., Snow, J. and Freise, M., (2004). Petrogenesis of oceanic plagiogranites by partial melting of gabbros: an experimental study. Contrib. Mineral. Petrol. 146, 414-432.

MacLeod, C. J., Dick, H. J. B., Blum, P., Abe, N., Blackman, D. K., Bowles, J. A., Cheadle, M. J., Cho, K., Ciazela, J., Deans, J. R., Edgcomb, V. P., Ferrando, C., France, L., Ghosh, B., Ildefonse, B. M., Kendrick, M. A., Koepke,J. H., Leong, J. A. M., Chuanzhou, L., Qiang, M., Morishita, T., Morris, A., Natland, J. H., Nozaka, T., Pluemper, O., Sanfilippo, A., Sylvan, J. B., Tivey, M. A., Tribuzio, R. and Viegas, L. G. F., (2017). Site U1473. Proc. ODP, Sci. Results,. 360, 136.

Müller, A., Wiedenbeck, M., Kerkhof, A., Kronz, A. and Simon, K., (2003). Trace elements in quartz - A combined electron microprobe, secondary ion mass spectrometry, laserablation ICP-MS, and cathodoluminescene study.
Nguyen, D. K., Morishita, T., Soda, Y., Tamura, A., Ghosh, B., Harigane, Y., France, L., Liu, C., Natland, J. H., Sanfilippo, A., MacLeod, C. J., Blum, P. and Dick, H. J. B., (2018). Occurrence of Felsic Rocks in Oceanic Gabbros from IODP Hole U1473A: Implications for Evolved Melt Migration in the Lower Oceanic Crust. Minerals. 8, 583.

Niu, Y., Gilmore, T., Mackie, S. M. and Bach, W., (2002). Mineral chemistry, whole-rock compositions, and petrogenesis of Leg 176 gabbros; data and discussion. Affili. 176, 60.

Philpotts, A. R., (1979). Silicate Liquid Immiscibility in Tholeiitic Basalts. J. Petrol. 20, 99-118.

Putnis, A., (2002). Mineral replacement reactions: From macroscopic observations to microscopic mechanisms. Mineral Mag. 66, 689-708.

Putnis, A., (2009). Mineral Replacement Reactions. Rev. Mineral. Geochem. 70, 87-124.

Putnis, A. and John, T., (2010). Replacement Processes in the Earth's Crust. Elements. 6, 159-164.

Putnis, A. and Putnis, C.V.,(2007).The mechanism of reequilibration of solids in the presence of a fluid phase. J. Solid State Chem. 180, 17831786.

W. Le Maitre, R., Streckeisen, A., Zanettin, B., Le Bas, M., Bonin, B. and Bateman, P. (2002) Igneous Rocks: A Classification and Glossary of Terms. Cambridge University Press. 